\title{
STOCHASTIC INTEGRALS AND CONDITIONAL FULL SUPPORT
}

\author{
MIKKO S. PAKKANEN, ${ }^{*}$ University of Helsinki
}

\begin{abstract}
We present conditions that imply the conditional full support (CFS) property, introduced in Guasoni, Rásonyi and Schachermayer (2008), for processes $Z:=H+\int K \mathrm{~d} W$, where $W$ is a Brownian motion, $H$ is a continuous process, and processes $H$ and $K$ are either progressive or independent of $W$. Moreover, in the latter case, under an additional assumption that $K$ is of finite variation, we present conditions under which $Z$ has CFS also when $W$ is replaced with a general continuous process with CFS. As applications of these results, we show that several stochastic volatility models and the solutions of certain stochastic differential equations have CFS.
\end{abstract}

Keywords: Conditional full support; stochastic integral; stochastic volatility; stochastic differential equation

2010 Mathematics Subject Classification: Primary 91B28

Secondary $60 \mathrm{H} 05$

\section{Introduction}

\subsection{Preliminaries}

The purpose of this paper is to show that certain stochastic integrals have the conditional full support (CFS) property, introduced in [10]. So, before stating the main results of this paper, let us recall the definition of this property.

To this end, recall first that when $E$ is a separable metric space and $\mu: \mathscr{B}(E) \rightarrow[0,1]$ is a Borel probability measure, the support of $\mu$, denoted by $\operatorname{supp}(\mu)$, is the (unique) minimal closed set $A \subset E$ such that $\mu(A)=1$. Let $\left(X_{t}\right)_{t \in[0, T]}$ be a continuous stochastic process taking values in an open interval $I \subset \mathbb{R}$, defined on a complete probability space $(\Omega, \mathcal{F}, \mathrm{P})$, and let $\mathbb{F}=\left(\mathcal{F}_{t}\right)_{t \in[0, T]}$ be a filtration on this space. Moreover, let $C_{x}([u, v], I)$ be the space of functions $f \in C([u, v], I)$ such that $f(u)=x \in I$. As usual, we equip the spaces $C([u, v], I)$ and $C_{x}([u, v], I), x \in I$, with the uniform topologies.

We say that the process $X$ has CFS with respect to the filtration $\mathbb{F}$, or briefly $\mathbb{F}$-CFS, if

(a) $X$ is adapted to $\mathbb{F}$,

(b) for all $t \in[0, T)$ and P-almost all $\omega \in \Omega$,

$$
\operatorname{supp}\left(\operatorname{law}\left[\left(X_{u}\right)_{u \in[t, T]} \mid \mathcal{F}_{t}\right](\omega)\right)=C_{X_{t}(\omega)}([t, T], I) .
$$

In (1.1), we regard law $\left[\left(X_{u}\right)_{u \in[t, T]} \mid \mathcal{F}_{t}\right]$ as a regular conditional law (a random Borel probability measure-see, e.g. [14, pp. 106-107]) on the space $C([t, T], I)$. Arguably, the formulation of the CFS property might appear slightly complicated at first sight, but informally it simply

Received 16 February 2009; revision received 26 April 2010.

* Postal address: Department of Mathematics and Statistics, University of Helsinki, PO Box 68, FI-00014 Helsingin yliopisto, Finland. Email address: msp@iki.fi 
means that when we observe $X$ from any time $t \in[0, T)$ onwards, $X$ still stays arbitrarily close to any continuous path in $I$ starting from $X_{t}$ with positive $\mathcal{F}_{t}$-conditional probability.

Throughout this paper, when we say that some process has CFS without specifying the filtration, we tacitly take it to be the natural filtration of the process. In fact, this is the "weakest form' of CFS that a process can have (see Corollary 2.1 and Lemma 2.3).

\subsection{Main results of the paper}

We shall establish CFS for processes of the form

$$
Z_{t}:=H_{t}+\int_{0}^{t} k_{s} \mathrm{~d} W_{s}, \quad t \in[0, T],
$$

where $H$ is a continuous process, the integrator $W$ is a Brownian motion, and the integrand $k$ satisfies some varying assumptions (to be clarified below).

We focus on three cases, each of which requires a separate treatment. First, we study the case in which

( $\left.\mathrm{Z}^{1}\right) H$ and $k$ are (jointly) independent of $W$.

We find that in this case, $Z$ has CFS if $t \mapsto k_{t}$ is almost everywhere nonvanishing almost surely (Theorem 3.1). As an application of this result, we show that several popular stochastic volatility models - which feature leverage effects, long memory in volatility, and volatility jumps-have the CFS property. Next, we relax the assumption about independence, and consider the case in which

$\left(\mathrm{Z}^{2}\right) H$ and $k$ depend progressively on $W$ and some additional continuous process that does not anticipate $W$, and $H=\int_{0}^{*} h_{s} \mathrm{~d} s$ is absolutely continuous.

It turns out that in this case the simple sufficient condition of the preceding case does not guarantee CFS - we present a very simple example, in which $k$ is strictly positive but $Z$ fails to have CFS. However, we show that, under a set of more stringent assumptions-which are satisfied, e.g. when $k$ is bounded from above and away from 0 , and $h$ is bounded - the process $Z$ does have CFS (Theorem 3.2). Using this result, we are able to establish CFS for the solutions of certain stochastic differential equations. Finally, we consider briefly the following (partial) extension of case $\left(Z^{1}\right)$.

( $\left.\mathrm{Z}^{3}\right)$ A (general) continuous process $X$ with CFS replaces $W$, processes $H$ and $k$ are (jointly) independent of $X$, and $k$ is of finite variation.

We show that in this case, $Z$ has CFS if each path of $k$ is bounded away from zero (Theorem 3.3).

\subsection{Motivation}

As mentioned before, the CFS property was first introduced by Guasoni et al. [10], in connection to mathematical finance, viz. pricing models with transaction costs. Their main result asserted that if a continuous price process has CFS then, for any $\varepsilon>0$, there exists a socalled $\varepsilon$-consistent price system, which is a martingale (after an equivalent change of measure) that shadows the price process within the bid-ask spread implied by $\varepsilon$-sized proportional transaction costs (see also [13] for a related study). The existence of $\varepsilon$-consistent price systems for all $\varepsilon>0$ implies that the price process does not admit arbitrage opportunities under arbitrary small transaction costs - since any arbitrage strategy would generate arbitrage also in the consistent price system, which is a contradiction because of the martingale property. 
Consistent price systems can be seen as generalizations of equivalent martingale measures (EMMs), since if a price process admits an EMM then the price process itself qualifies as a trivial $\varepsilon$-consistent price system for any $\varepsilon>0$.

CFS is however worth studying even when it comes to price processes that admit EMMs, since it enables the construction of specific consistent price systems that are useful in solving superreplication problems under proportional transaction costs. This is manifested by the 'face-lifting' result in [10], which says that if $\left(P_{t}\right)_{t \in[0, T]}$ is a price process with CFS then the superreplication price of any European-style vanilla contingent claim $g\left(P_{T}\right)$ under $\varepsilon$-sized proportional transaction costs tends to $\hat{g}\left(P_{0}\right)$ when $\varepsilon \downarrow 0$, where $\hat{g}$ is the concave envelope of $g$ (the smallest concave function that majorizes $g$ ). This means that superreplicating, e.g. a European call option under small proportional transaction costs, entails buying the underlying, the trivial superreplicating portfolio.

In addition to models with transaction costs, CFS has also been found to have relevance with frictionless pricing models, as indicated in the recent paper of Bender et al. [2]. Their result asserted that if a continuous price process has CFS (they used the name conditional small ball property) and pathwise quadratic variation, then it does not admit arbitrage opportunities in a class of trading strategies that is somewhat narrower than what the classical models allow, but nevertheless covers a large share (if not almost all) of the strategies that have practical relevance.

Aside from having these applications in mathematical finance, CFS is an interesting fundamental property from a purely mathematical point of view. In particular, research on the CFS property can be seen as a natural continuation to the classical studies of the supports of the laws of continuous Gaussian processes by Kallianpur [15], and diffusions, initiated by Stroock and Varadhan [20] and continued by several other authors (see, e.g. [16] and the references therein).

\subsection{Previous results}

So far, a variety of continuous processes, which are nondegenerate in some sense, have been shown to have CFS.

Gaussian processes that have CFS include fractional Brownian motion, with any Hurst index $\mathfrak{h} \in(0,1)$ (and specifically when $\mathfrak{h}=\frac{1}{2}$, the standard Brownian motion) [10], and more generally all Brownian moving averages with nonvanishing kernels [4]. Moreover, Gaussian processes with stationary increments that satisfy a certain spectral density condition have CFS [9].

In the case of continuous Markov processes, showing CFS reduces to showing that the support of the (unconditional) law of the process is the largest possible for any initial condition, as pointed out in [10]. Hence, the classical results concerning the supports of diffusions (see, e.g. [16] and [20]) can be used to establish CFS.

Moreover, it was shown in [10] that if a continuous process $X$ has CFS then the Riemann integral process $\int_{0}^{\cdot} X_{t} \mathrm{~d} t$ has CFS, which allows (using iteration) construction of processes that have CFS and arbitrarily smooth paths.

While many examples of common Gaussian and Markovian processes have CFS, this observation should not be extrapolated too much, since CFS is a not a trivial property, due to its functional nature. For example, nondegeneracy of finite-dimensional conditional laws does not typically, even in the case of Gaussian processes, guarantee CFS. A striking example of this difficulty is the continuous Gaussian process $\left(X_{t}\right)_{t \in[0,1]}$ constructed by Cherny [4, Example 3.1], which satisfies $\operatorname{var}\left[X_{t} \mid \mathcal{F}_{S}^{X}\right]>0$ almost surely (a.s.) for all $t, s \in[0,1]$ such that $t>s$, but nevertheless $\int_{0}^{1} X_{t} \mathrm{~d} t=0$ a.s.-implying that $X$ cannot have CFS. 


\subsection{Outline of the paper}

Section 2 contains some basic results on the CFS property, to familiarize the reader with the property, and to facilitate subsequent proofs. Section 3 contains the main results concerning stochastic integrals. Finally, Section 4 concludes with applications to the aforementioned more specific processes: stochastic volatility models and solutions of stochastic differential equations.

\subsection{Notation and conventions}

Let $\mathbb{T} \subset[0, \infty)$ be a left-closed interval, and let $\left(X_{t}\right)_{t \in \mathbb{T}}$ be a generic stochastic process on $(\Omega, \mathcal{F}, \mathrm{P})$. We say that $X$ is jointly measurable if $(t, \omega) \mapsto X_{t}(\omega)$ is measurable with respect to $\mathcal{B}(\mathbb{T}) \otimes \mathcal{F}$. Throughout this paper, we implicitly assume that all processes are jointly measurable, whenever this is not already implied by continuity (from left or right) or progressive measurability. For any $t \in \mathbb{T}$, we write $\hat{X}^{t}:=\left(X_{s}-X_{t}\right)_{s \geq t}$.

We denote by $\tilde{\mathbb{F}}^{X}=\left(\tilde{\mathcal{F}}_{t}^{X}\right)_{t \in \mathbb{T}}$ the 'raw' natural filtration of $X$ and by $\mathbb{F}^{X}=\left(\mathcal{F}_{t}^{X}\right)_{t \in \mathbb{T}}$ its usual augmentation (the minimal right-continuous augmentation of $\tilde{\mathbb{F}}^{X}$ such that $\mathcal{F}_{\min \mathbb{T}}^{X}$ contains all P-null sets in $\tilde{\mathcal{F}}_{t}^{X}$ for all $t \in \mathbb{T}$; see, e.g. [17, p. 45]).

As usual, $\|\cdot\|_{\infty}$ denotes the sup-norm, and, for any $f, g \in C(\mathbb{T}):=C(\mathbb{T}, \mathbb{R})$ and $r>0$, write $B(g, r):=\left\{h \in C(\mathbb{T}):\|h-g\|_{\infty}<r\right\}$ and $I(f, g, r):=\mathbf{1}_{B(g, r)}(f)$.

Finally, $\mathbb{R}_{+}:=(0, \infty), \mathbb{Q}_{+}:=\mathbb{Q} \cap \mathbb{R}_{+}, \mathbb{N}:=\{0,1, \ldots\}, \mathbb{Z}_{+}:=\mathbb{N} \backslash\{0\}$, and $\lambda$ stands for the Lebesgue measure on $\mathbb{R}$.

\section{Basic results on the CFS property}

Since CFS is a very recent concept, in the absence of any comprehensive account, it is instructive to present a few basic results that can be used to establish the property. We will consider processes and their CFS in the largest possible state space $\mathbb{R}$, but this is not really a restriction, since all of the following results, except Lemma 2.1, can also be applied to processes in smaller state spaces using the following observation.

Remark 2.1. If $I \subset \mathbb{R}$ is an open interval and $f: \mathbb{R} \rightarrow I$ is a homeomorphism, then $g \mapsto f \circ g$ is a homeomorphism between $C_{x}([0, T])$ and $C_{f(x)}([0, T], I)$. Hence, for $f(X)$, understood as a process in $I$, we have

$$
f(X) \text { has } \mathbb{F} \text {-CFS } \Longleftrightarrow \quad X \text { has } \mathbb{F} \text {-CFS. }
$$

We begin with an alternative 'small-ball' characterization of CFS, which is more tractable than the original definition (1.1).

Lemma 2.1. (Small-ball probabilities.) Let $\left(X_{t}\right)_{t \in[0, T]}$ be a continuous process, adapted to filtration $\mathbb{F}=\left(\mathcal{F}_{t}\right)_{t \in[0, T]}$. Then, $X$ has $\mathbb{F}-C F S$ if and only if

$$
\mathrm{E}\left[I\left(\hat{X}^{t}, f, \varepsilon\right) \mid \mathcal{F}_{t}\right]>0 \text { a.s. }
$$

for all $t \in[0, T), f \in C_{0}([t, T])$, and $\varepsilon>0$.

Proof. Let $t \in[0, T)$ be fixed. For brevity, define $\mu_{\omega}:=\operatorname{law}\left[\left(X_{u}\right)_{u \in[t, T]} \mid \mathcal{F}_{t}\right](\omega)$ and $\hat{\mu}_{\omega}:=\operatorname{law}\left[\hat{X}^{t} \mid \mathcal{F}_{t}\right](\omega)$ for all $\omega \in \Omega$. It is straightforward to check that

$$
\operatorname{supp}\left(\hat{\mu}_{\omega}\right)=C_{0}([t, T]) \Longleftrightarrow \operatorname{supp}\left(\mu_{\omega}\right)=C_{X_{t}(\omega)}([t, T])
$$

for almost all (a.a.) $\omega \in \Omega$. The space $C_{0}([t, T])$ is separable (e.g. by the Stone-Weierstrass 
theorem), so there exists a countable dense family $\left\{f_{n}: n \in \mathbb{N}\right\} \subset C_{0}([t, T])$. Hence, the equality on the left-hand side of (2.3) holds if and only if $\hat{\mu}_{\omega}\left(B\left(f_{n}, q\right)\right)>0$ for all $n \in \mathbb{N}$ and $q \in \mathbb{Q}_{+}$. By virtue of countability, we find that $\mathrm{P}\left[\operatorname{supp}(\hat{\mu})=.C_{0}([t, T])\right]=1$ if and only if

$$
\mathrm{P}\left[\hat{\mu} .\left(B\left(f_{n}, q\right)\right)>0\right]=1 \quad \text { for all } n \in \mathbb{N} \text { and } q \in \mathbb{Q}_{+} .
$$

By the disintegration theorem (see Theorem 6.4 of [14]), we have

$$
\hat{\mu} .\left(B\left(f_{n}, q\right)\right)=\mathrm{E}\left[I\left(\hat{X}^{t}, f_{n}, q\right) \mid \mathcal{F}_{t}\right] \quad \text { a.s., }
$$

so (2.4) is clearly equivalent to the asserted condition (2.2).

Remark 2.2. While Lemma 2.1 is somewhat obvious, it has two very important consequences. Firstly, we note that the characterization (2.2) is stated in terms of conditional expectations. Hence, CFS does not hinge on any particular choice of versions of the regular conditional laws. Secondly, whenever we want to argue contrapositively and assume that the CFS property fails to hold, Lemma 2.1 guarantees that there exists a fixed ball $B(f, \varepsilon)$ such that $\hat{X}^{t}$ exits $B(f, \varepsilon)$ with positive probability. Ignoring separability, the definition of CFS alone would then only imply existence of a random ball $B(f(\omega), \varepsilon(\omega))$ with the same property, which would cause certain complications (primarily, the need to find a measurable selection of these balls).

Thus, establishing CFS reduces to checking that certain conditional expectations are positive. It is sometimes easier to show positivity of a conditional expectation by arguing that the analogous conditional expectation with respect to some larger $\sigma$-algebra is positive, and then pass to the original $\sigma$-algebra using the following elementary fact.

Lemma 2.2. (Positivity.) Let $\mathcal{g}$ and $\mathscr{H}$ be $\sigma$-algebras such that $\mathcal{G} \subset \mathcal{H}$, and let $Y \in L^{1}$ such that $Y \geq 0$. If $\mathrm{E}[Y \mid \mathcal{H}]>0$ a.s. then $\mathrm{E}[Y \mid \mathcal{G}]>0$ a.s.

Combining Lemmas 2.1 and 2.2 we find that, like the semimartingale property, CFS is preserved when the filtration shrinks, as long as the process is adapted to the smaller filtration (this observation is not really new, it was already employed, e.g. in [2] and [4]).

Corollary 2.1. (Smaller filtration.) Let $\left(X_{t}\right)_{t \in[0, T]}$ be a continuous process, adapted to filtrations $\mathbb{F}=\left(\mathcal{F}_{t}\right)_{t \in[0, T]}$ and $\mathbb{G}=\left(g_{t}\right)_{t \in[0, T]}$ that satisfy $g_{t} \subset \mathcal{F}_{t}$ for all $t \in[0, T]$. Then, $X$ has $\mathbb{G}-C F S$ if it has $\mathbb{F}-C F S$.

Next we shall show a result to the opposite direction, namely that CFS is preserved when the filtration is augmented the usual way (see, e.g. [17, p. 45]).

Lemma 2.3. (Usual augmentation.) Let $\left(X_{t}\right)_{t \in[0, T]}$ be a continuous process, adapted to filtration $\mathbb{F}=\left(\mathcal{F}_{t}\right)_{t \in[0, T]}$. Then, $X$ has $\mathbb{F}-C F S$ if and only if it has CFS with respect to the usual augmentation of $\mathbb{F}$.

Proof. The 'if' part follows from Corollary 2.1. Moreover, regarding the 'only if' part, it follows from a simple monotone class argument that adding the P-null sets in $\mathcal{F}_{T}$ to the filtration does not alter conditional expectations, and, hence, by Lemma 2.1, CFS remains intact. Thus, it suffices to show that passing to the right-continuous augmentation $\left(\mathcal{F}_{t+}\right)_{t \in[0, T]}$ preserves CFS.

To this end, we shall argue contrapositively, that if CFS with respect to $\left(\mathcal{F}_{t+}\right)_{t \in[0, T]}$ fails at time $\underline{t}$ then CFS with respect to $\mathbb{F}$ must fail at some time $\underline{t}+\delta$, where $\delta>0$ is small. So, when $X$ does not have $\left(\mathcal{F}_{t+}\right)_{t \in[0, T]}$-CFS, by Lemma 2.1 , there exist $\underline{t} \in[0, T), f \in C_{0}([\underline{t}, T])$, and $\varepsilon>0$ such that $\mathrm{P}[A]>0$, where $A:=\left\{\mathrm{E}\left[I\left(\hat{X}^{t}, f, \varepsilon\right) \mid \mathcal{F}_{\underline{t}+}\right]=0\right\}$. Define

$$
\tau:=\inf \left\{t \in(\underline{t}, T]:\left|X_{t}-X_{\underline{t}}-f(t)\right| \geq \varepsilon\right\},
$$




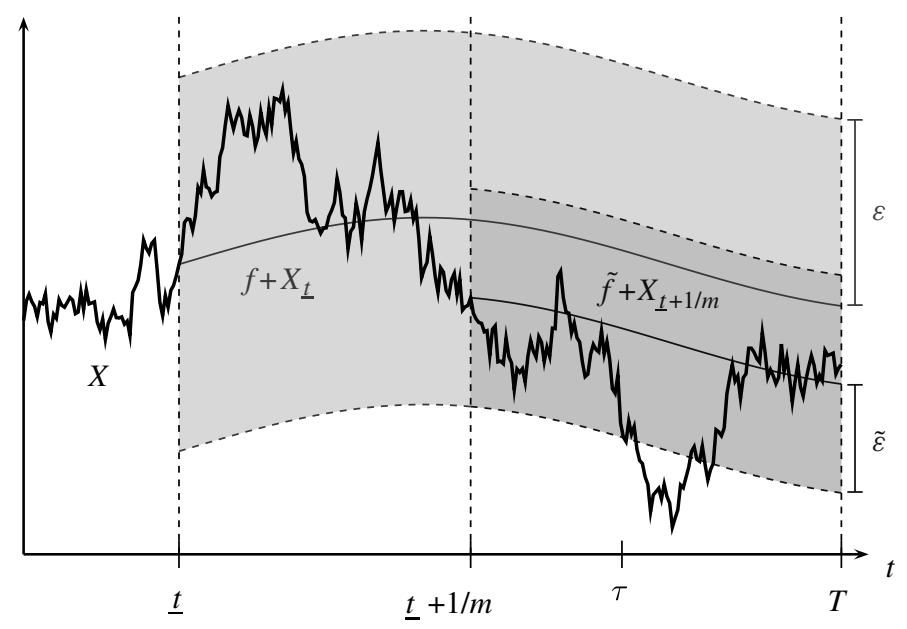

FIGURE 1: Choosing $\tilde{f}$ and $\tilde{\varepsilon}$ in the proof of Lemma 2.3.

where inf $\varnothing:=\infty$ by convention. Obviously, $\mathrm{E}\left[\mathbf{1}_{\{\tau<\infty\}} \mid \mathcal{F}_{t+}\right]=1$ almost everywhere (a.e.) on $A$. For any $n \in \mathbb{Z}_{+}$, let us define $A_{n}:=\{\tau>\underline{t}+1 / n\} \cap A$, and note that $A_{n} \in \mathscr{F}_{\underline{t}+1 / n}$, since $\tau$ is a stopping time with respect to $\left(\mathcal{F}_{t}\right)_{t \in[t, T]}$, and since $A \in \mathscr{F}_{t+} \subset \mathscr{F}_{t+1 / n}$. Moreover, since $A_{n} \uparrow A$, we have $\mathrm{P}\left[A_{m}\right]>0$ for some $m \in \mathbb{Z}_{+}$such that $\underline{t}+1 / m<T$. Clearly,

$$
\mathrm{E}\left[\mathbf{1}_{A} \mathrm{E}\left[\mathbf{1}_{\{\tau<\infty\}} \mid \mathscr{F}_{\underline{t}+1 / m}\right]\right]=\mathrm{E}\left[\mathbf{1}_{A} \mathbf{1}_{\{\tau<\infty\}}\right]=\mathrm{E}\left[\mathbf{1}_{A} \mathrm{E}\left[\mathbf{1}_{\{\tau<\infty\}} \mid \mathscr{F}_{\underline{t}+}\right]\right]=\mathrm{P}[A],
$$

which implies that $\mathrm{E}\left[\mathbf{1}_{\{\tau<\infty\}} \mid \mathcal{F}_{\underline{t}+1 / m}\right]=1$ a.e. on $A$. Furthermore, using inclusions $A_{m} \subset$ $\{\tau>\underline{t}+1 / m\}$ and $A_{m} \subset A$, we see that

$$
\mathrm{E}\left[\mathbf{1}_{\{\underline{t}+1 / m<\tau<\infty\}} \mid \mathcal{F}_{\underline{t}+1 / m}\right]=\mathrm{E}\left[\mathbf{1}_{\{\tau<\infty\}} \mid \mathcal{F}_{\underline{t}+1 / m}\right]=1 \text { a.e. on } A_{m} .
$$

Now, define

$$
\begin{gathered}
\tilde{f}(t):=f(t)-f\left(\underline{t}+\frac{1}{m}\right), \quad t \in[\underline{t}+1 / m, T], \\
\tilde{\varepsilon}(\omega):=\varepsilon-\left|X_{\underline{t}+1 / m}(\omega)-X_{\underline{t}}(\omega)-f\left(\underline{t}+\frac{1}{m}\right)\right|
\end{gathered}
$$

(see Figure 1), and note that, by (2.5) we have $\tilde{\varepsilon}>0$ a.e. on $A_{m}$. Moreover,

$$
\begin{aligned}
\left\{\underline{t}+\frac{1}{m}<\tau<\infty\right\} & =\left\{\sup _{t \in(\underline{t}+1 / m, T]}\left|X_{t}-X_{\underline{t}}-f(t)\right| \geq \varepsilon\right\} \\
& \subset\left\{\sup _{t \in(\underline{t}+1 / m, T]}\left|X_{t}-X_{\underline{t}+1 / m}-\tilde{f}(t)\right| \geq \tilde{\varepsilon}\right\} \\
& =: F
\end{aligned}
$$

by the triangle inequality. For all $n \in \mathbb{Z}_{+}$, define $B_{n}:=A_{m} \cap\{\tilde{\varepsilon}>1 / n\} \in \mathcal{F}_{\underline{t}+1 / m}$. Since $B_{n} \uparrow A_{m} \cap\{\tilde{\varepsilon}>0\}$ and $\mathrm{P}\left[A_{m} \cap\{\tilde{\varepsilon}>0\}\right]=\mathrm{P}\left[A_{m}\right]>0$, we have $\mathrm{P}\left[B_{m^{\prime}}\right]>0$ for some $m^{\prime} \in \mathbb{Z}_{+}$. Using (2.5) and (2.6), we find that

$$
\mathrm{E}\left[I\left(\hat{X}^{t+1 / m}, \tilde{f}, \frac{1}{m^{\prime}}\right) \mid \mathcal{F}_{\underline{t}+1 / m}\right] \leq 1-\mathrm{E}\left[\mathbf{1}_{\left\{\tilde{\varepsilon}>1 / m^{\prime}\right\} \cap F} \mid \mathscr{F}_{\underline{t}+1 / m}\right]=0 \text { a.e. on } B_{m^{\prime}} .
$$

Thus, by Lemma 2.1, $X$ does not have $\mathbb{F}$-CFS, which concludes the proof. 
We conclude this section by showing that CFS - with respect to the natural filtration of the process - is a property of the law of the process, and thus does not depend on the underlying probability space. This allows for flexibility in the subsequent proofs, where it is necessary to assume that the probability space enjoys some specific properties.

Lemma 2.4. (Law invariance.) Let $\left(X_{t}\right)_{t \in[0, T]}$ and $\left(Y_{t}\right)_{t \in[0, T]}$ be continuous processes (possibly defined on distinct probability spaces) such that $X \stackrel{\mathrm{LAW}}{=} Y$. Then, $X$ has $\mathbb{F}^{X}-C F S$ if and only if $Y$ has $\mathbb{F}^{Y}-C F S$.

Proof. By Lemma 2.3, it suffices to show the equivalence with respect to 'raw' natural filtrations. Moreover, it is clearly not a restriction to assume that $X$ and $Y$ are defined on the same probability space. Let $t \in[0, T), f \in C_{0}([t, T])$, and $\varepsilon>0$. Denote by $\left\{t_{k}: k \in \mathbb{N}\right\}$ some enumeration of $[0, t] \cap \mathbb{Q}$. By continuity of paths and Theorem 7.23 of [14], we have

$$
\mathrm{E}\left[I\left(\hat{X}^{t}, f, \varepsilon\right) \mid X_{t_{1}}, \ldots X_{t_{n}}\right] \rightarrow \mathrm{E}\left[I\left(\hat{X}^{t}, f, \varepsilon\right) \mid \tilde{\mathcal{F}}_{t}^{X}\right]
$$

and

$$
\mathrm{E}\left[I\left(\hat{Y}^{t}, f, \varepsilon\right) \mid Y_{t_{1}}, \ldots Y_{t_{n}}\right] \rightarrow \mathrm{E}\left[I\left(\hat{Y}^{t}, f, \varepsilon\right) \mid \tilde{\mathcal{F}}_{t}^{Y}\right]
$$

a.s. when $n \rightarrow \infty$. On the other hand, $X \stackrel{\text { LAW }}{=} Y$ and Exercise 6.12 of [14] imply that

$$
\mathrm{E}\left[I\left(\hat{X}^{t}, f, \varepsilon\right) \mid X_{t_{1}}, \ldots X_{t_{n}}\right] \stackrel{\mathrm{LAW}}{=} \mathrm{E}\left[I\left(\hat{Y}^{t}, f, \varepsilon\right) \mid Y_{t_{1}}, \ldots Y_{t_{n}}\right]
$$

for all $n \in \mathbb{N}$. Since equality in law holds also in the limit, the assertion follows from Lemma 2.1.

\section{CFS for stochastic integrals}

\subsection{Independent integrands and Brownian integrators}

We shall now move to the main results of this paper. First we establish CFS for the process $Z$, as defined by (1.2), in the case $\left(Z^{1}\right)$.

Theorem 3.1. (CFS.) Let $\left(H_{t}\right)_{t \in[0, T]}$ be a continuous process, let $\left(k_{t}\right)_{t \in[0, T]}$ be a process such that $\int_{0}^{T} k_{s}^{2} \mathrm{~d} s<\infty$ a.s., and let $\left(W_{t}\right)_{t \in[0, T]}$ be a Brownian motion independent of $(H, k)$. If

$$
\lambda\left(\left\{t \in[0, T]: k_{t}=0\right\}\right)=0 \text { a.s. }
$$

then the process

$$
Z_{t}:=H_{t}+\int_{0}^{t} k_{s} \mathrm{~d} W_{s}, \quad t \in[0, T],
$$

has CFS.

Remark 3.1. It follows from Fubini's theorem that if $k_{t} \neq 0$ a.s. for all $t \in[0, T]$ then condition (3.1) holds. Hence, in particular, whenever $k_{t}$ has continuous distribution for all $t$, Theorem 3.1 applies.

Remark 3.2. The process $Z$ does not have to be a semimartingale, as $H$ is only assumed to be continuous. To give a nontrivial example, if

$$
H_{t}:=B_{t}^{\mathfrak{h}}, \quad k_{t}:=1, \quad t \in[0, T],
$$

where $B^{\mathfrak{h}}$ is a fractional Brownian motion, independent of $W$, with Hurst index $\mathfrak{h} \in(0,1)$, then $Z$ is a mixed fractional Brownian motion, which is not a semimartingale when $\mathfrak{h} \in\left(0, \frac{1}{2}\right) \cup\left(\frac{1}{2}, \frac{3}{4}\right)$ (as shown in [3]). 
The proof of Theorem 3.1 requires some preparation. Specifically, we shall show that the Wiener integral of an a.e. nonvanishing function has positive small-ball probabilities, using a time-change argument similar to the one that appears in [19].

Lemma 3.1. (Wiener integrals.) Let $h \in C([0, T])$ and $k \in L^{2}([0, T])$, let $\left(W_{t}\right)_{t \in[0, T]}$ be a Brownian motion, and define

$$
J_{t}:=h(t)+\int_{0}^{t} k(s) \mathrm{d} W_{s}, \quad t \in[0, T] .
$$

If $k(t) \neq 0$ for a.a. $t \in[0, T]$ then, for all $\underline{t} \in[0, T), f \in C_{0}([\underline{t}, T])$, and $\varepsilon>0$, we have

$$
\mathrm{P}\left[\sup _{t \in[\underline{t}, T]}\left|J_{t}-J_{\underline{t}}-f(t)\right|<\varepsilon\right]>0 .
$$

Proof. Clearly, we may assume that $h=0$. Let $\underline{t} \in[0, T), f \in C_{0}([\underline{t}, T])$, and $\varepsilon>0$. Define

$$
g(t):=\int_{\underline{t}}^{t} \mathrm{~d}\langle J, J\rangle_{u}=\int_{\underline{t}}^{t} k(s)^{2} \mathrm{~d} s, \quad t \in[\underline{t}, T],
$$

and note that since $k(t) \neq 0$ for a.a. $t \in[0, T], g$ is a homeomorphism between $[\underline{t}, T]$ and $[0, K]$, where $K:=\int_{t}^{T} k(s)^{2} \mathrm{~d} s$. By the Dambis, Dubins-Schwarz theorem, there exists a Brownian motion $\left(B_{S}\right)_{s \in[0, K]}^{-}$such that $J_{t}-J_{\underline{t}}=B_{g(t)}, t \in[\underline{t}, T]$, a.s. Hence, we obtain

$$
\begin{aligned}
\sup _{t \in[\underline{t}, T]}\left|J_{t}-J_{\underline{t}}-f(t)\right| & =\sup _{t \in[\underline{t}, T]}\left|B_{g(t)}-\left(f \circ g^{-1}\right)(g(t))\right| \\
& =\sup _{u \in[0, K]}\left|B_{u}-\left(f \circ g^{-1}\right)(u)\right| \text { a.s. }
\end{aligned}
$$

Since $f \circ g^{-1}$ is continuous, and since the Wiener measure is supported on $C_{0}([0, K])$ (see, e.g. Corollary VIII.2.3 of [17]), we have

$$
\mathrm{P}\left[\sup _{t \in[\underline{t}, T]}\left|J_{t}-J_{\underline{t}}-f(t)\right|<\varepsilon\right]=\mathrm{P}\left[\sup _{s \in[0, K]}\left|B_{S}-\left(f \circ g^{-1}\right)(s)\right|<\varepsilon\right]>0 .
$$

We shall now deduce Theorem 3.1 from Lemma 3.1 using a suitable conditioning scheme.

Proof of Theorem 3.1 (beginning). Let $\underline{t} \in[0, T), f \in C_{0}([\underline{t}, T])$, and $\varepsilon>0$. Furthermore, let $(\Omega, \mathcal{F}, \mathrm{P})$ be the completed probability space that carries $W, H$, and $k$. By Lemma 2.1, it suffices to show that

$$
\mathrm{E}\left[I\left(\hat{Z}^{t}, f, \varepsilon\right) \mid \tilde{\mathcal{F}}_{\underline{t}}^{Z}\right]>0 \quad \text { P-a.s. }
$$

The proof of this assertion becomes more transparent when we work on an extension of the space $(\Omega, \mathcal{F}, \mathrm{P})$. Namely, we show an analogous property for a variant of $Z$, denoted by $Z^{\star}$, in which the integrator is $W$ up to time $\underline{t}$, but further Brownian increments of the integrator are defined on an auxiliary space. Then, since $Z$ and $Z^{\star}$ have the same distribution (e.g. by Exercise IV.5.16 of [17]), it follows that (3.2) holds, by the argument used in the proof of Lemma 2.4.

We define the extended space by

$$
\Omega^{\star}:=\Omega \times C_{0}([0, T]), \quad \mathscr{F}^{\star}:=\overline{\mathscr{F} \otimes \mathscr{B}\left(C_{0}([0, T])\right)}, \quad \mathrm{P}^{\star}:=\overline{\mathrm{P} \otimes \nu},
$$


where $v$ is the Wiener measure on $C_{0}([0, T])$ and the bars denote completion. For any $\omega^{\star}=$ $\left(\omega, \omega^{\prime}\right) \in \Omega^{\star}$, we define $B_{t}\left(\omega^{\star}\right):=B_{t}\left(\omega^{\prime}\right):=\omega^{\prime}(t)$ and $W_{t}^{\star}\left(\omega^{\star}\right):=B_{t \vee \underline{t}}\left(\omega^{\prime}\right)-B_{\underline{t}}\left(\omega^{\prime}\right)+$ $W_{t \wedge t}(\omega)$ for all $t \in[0, T]$. Moreover, we denote by $\mathrm{E}^{\star}$ the expectation with respect to $\mathrm{P}^{\star}$, by $\bar{X}$ the identity map on $\Omega$, which can be seen as a random element in the measurable space $(\Omega, \mathcal{F})$, and by $Z^{\star}$ the process analogous to $Z$, with $W^{\star}$ as the integrator. Note that by joint measurability we have $H_{t}(\omega)=\phi(t, \omega)$ and $k_{t}(\omega)=\psi(t, \omega)$ for some $(\mathscr{B}([0, T]) \otimes \mathcal{F})$ measurable functions $\phi$ and $\psi$ from $[0, T] \times \Omega$ to $\mathbb{R}$.

For the conclusion of the proof, we need the following auxiliary result, which asserts that 'freezing' randomness on the original probability space $\Omega$ reduces $Z^{\star}$ to a Wiener integral with a drift.

Lemma 3.2. (Freezing.) For P-a.a. $\omega \in \Omega$, we have

$$
\left(\hat{Z}_{t}^{\star, \underline{t}}(\omega, \cdot)\right)_{t \in[\underline{t}, T]}=\left(\phi(t, \omega)-\phi(\underline{t}, \omega)+\int_{\underline{t}}^{t} \psi(s, \omega) \mathrm{d} B_{s}\right)_{t \in[t, T]}
$$

up to $v$-indistinguishability, where the integral on the right-hand side is a Wiener integral.

Proof. Let us denote by $J^{\omega}$ the process on the right-hand side of (3.3). By a standard argument (see, e.g. Lemma 17.23 of [14]), there exist measurable functions $\psi_{n}, n \in \mathbb{N}$, from $[\underline{t}, T] \times \Omega$ to $\mathbb{R}$ such that

$$
\psi_{n}(t, \omega)=\sum_{i=1}^{k_{n}} \xi_{i}^{n}(\omega) \mathbf{1}_{\left(t_{i}^{n}, t_{i+1}^{n}\right]}(t) \quad \text { and } \quad\left\|\psi_{n}(\cdot, \omega)-\psi(\cdot, \omega)\right\|_{L^{2}[t, T]} \rightarrow 0
$$

when $n \rightarrow \infty$ for P-a.a. $\omega \in \Omega$. Now define

$$
\begin{aligned}
I_{t}^{n}\left(\omega, \omega^{\prime}\right) & :=\phi(t, \omega)+\phi(\underline{t}, \omega)+\int_{\underline{t}}^{t} \psi_{n}(s, \omega) \mathrm{d} W_{s}^{\star}\left(\omega, \omega^{\prime}\right) \\
& =\phi(t, \omega)+\phi(0, \omega)+\sum_{i=1}^{k_{n}} \xi_{i}^{n}(\omega)\left(B_{t_{i+1}^{n} \wedge t}\left(\omega^{\prime}\right)-B_{t_{i}^{n} \wedge t}\left(\omega^{\prime}\right)\right) .
\end{aligned}
$$

By $L^{2}$-continuity of Itô integrals and the Borel-Cantelli lemma, there exists a sequence $n_{m} \uparrow \infty$ such that $\sup _{t \in[\underline{t}, T]}\left|\hat{Z}_{t}^{\star, t}-I_{t}^{n_{m}}\right| \rightarrow 0, \mathrm{P}^{\star}$-a.s. when $m \rightarrow \infty$. Hence, by Fubini's theorem,

$$
\sup _{t \in[\underline{t}, T]}\left|\hat{Z}_{t}^{\star, \underline{t}}(\omega, \cdot)-I_{t}^{n_{m}}(\omega, \cdot)\right| \rightarrow 0 \quad v \text {-a.s. }
$$

for P-a.a. $\omega \in \Omega$. On the other hand, $L^{2}$-continuity of Wiener integrals implies that, for P-a.a. $\omega \in \Omega$, also

$$
\sup _{t \in[\underline{t}, T]}\left|J_{t}^{\omega}-I_{t}^{n_{m}}(\omega, \cdot)\right| \rightarrow 0 \quad \text { in } v \text {-probability. }
$$

Now, since we have, for P-a.a. $\omega \in \Omega$,

$$
\begin{aligned}
& \mathrm{E}^{\nu}\left[\sup _{t \in[\underline{\underline{t}, T]}}\left|J_{t}^{\omega}-\hat{Z}_{t}^{\star, \underline{t}}(\omega, \cdot)\right| \wedge 1\right] \leq \mathrm{E}^{\nu}\left[\sup _{t \in[\underline{t}, T]}\left|\hat{Z}_{t}^{\star, \underline{t}}(\omega, \cdot)-I_{t}^{n_{m}}(\omega, \cdot)\right| \wedge 1\right] \\
& +\mathrm{E}^{v}\left[\sup _{t \in[t, T]}\left|J_{t}^{\omega}-I_{t}^{n_{m}}(\omega, \cdot)\right| \wedge 1\right] \text {, }
\end{aligned}
$$

letting $m \rightarrow \infty$ completes the proof. 
Proof of Theorem 3.1 (conclusion). Let us define

$$
g:=\mathcal{F} \otimes\left\{\varnothing, C_{0}([0, T])\right\}
$$

We shall show that $\mathrm{E}^{\star}\left[I\left(\hat{Z}^{\star, \underline{t}}, f, \varepsilon\right) \mid g\right]>0, \mathrm{P}^{\star}$-a.s., which, by Lemma 2.2 , implies that the same also holds with respect to $\tilde{\mathcal{F}}_{t} Z^{\star} \subset \mathcal{g}$, which in turn implies that (3.2) holds. We may compose

$$
\hat{Z}^{\star, \underline{t}}\left(\omega, \omega^{\prime}\right)=\hat{Z}^{\star, \underline{t}}\left(X(\omega), B\left(\omega^{\prime}\right)\right), \quad\left(\omega, \omega^{\prime}\right) \in \Omega^{\star} .
$$

Moreover, by independence, $v$ is a version of the regular $g$-conditional law of $B$ on $C_{0}([0, T])$. By the disintegration theorem (see Theorem 6.4 of [14]), we have, $\mathrm{P}^{\star}$-a.s.,

$$
\begin{aligned}
\mathrm{E}^{\star}\left[I\left(\hat{Z}^{\star, t}, f, \varepsilon\right) \mid g\right] & =\mathrm{E}^{\star}\left[\mathbf{1}_{B(f, \varepsilon)}\left(\hat{Z}^{\star, t}(X, B)\right) \mid g\right] \\
& =\int_{C_{0}([0, T])} \mathbf{1}_{B(f, \varepsilon)}\left(\hat{Z}^{\star,-t}\left(X, \omega^{\prime}\right)\right) v\left(\mathrm{~d} \omega^{\prime}\right) \\
& =: Y(X) .
\end{aligned}
$$

By Lemma 3.2, for P-a.a. $\omega \in \Omega$,

$$
\mathbf{1}_{B(f, \varepsilon)}\left(\hat{Z}^{\star, t}(\omega, \cdot)\right)=\mathbf{1}_{B(f, \varepsilon)}\left(J^{\omega}\right) \quad v \text {-a.s. }
$$

where $J^{\omega}$ is the right-hand side of (3.3). But, for P-a.a. $\omega \in \Omega$, the map $\psi(\cdot, \omega)$ is a.e. nonvanishing, so it follows from Lemma 3.1 that, for P-a.a. $\omega \in \Omega$,

$$
Y(X(\omega))=\int_{C_{0}([0, T])} \mathbf{1}_{B(f, \varepsilon)}\left(J^{\omega}\left(\omega^{\prime}\right)\right) v\left(\mathrm{~d} \omega^{\prime}\right)>0 .
$$

Hence, $Y>0$ also $\mathrm{P}^{\star}$-a.s., which concludes the proof.

\subsection{Progressively measurable integrands and Brownian integrators}

In case $\left(\mathrm{Z}^{1}\right),(H, k)$ and $W$ were assumed to be independent. We shall now move to case $\left(\mathrm{Z}^{2}\right)$ and dispense with this assumption. Before stating the result, let us consider two examples that motivate why the conditions we now impose on $H$ and $k$ need to be-apart from allowing dependence-more stringent than earlier.

Example 3.1. When the integrand $k$ is allowed to depend on the Brownian motion $W$, condition (3.1) is no longer sufficient. Namely, if we set e.g.

$$
H_{t}:=1, \quad k_{t}:=\mathrm{e}^{W_{t}-t / 2}, \quad t \in[0, T],
$$

then $Z=k=\mathcal{E}(W)$, the Doléans exponential of $W$, which is strictly positive and thus does not have CFS, when understood as a process in $\mathbb{R}$.

Example 3.2. Even when $k$ is positive and constant, but $H$ depends on $W, Z$ might not have CFS. To demonstrate this, let $\left(B_{t}\right)_{t \in[0, T]}$ be a Brownian motion and let $\mathbb{G}=\left(g_{t}\right)_{t \in[0, T]}$ be a filtration defined by

$$
g_{t}:=\bigcap_{s>t}\left(\mathcal{F}_{s}^{B} \vee \sigma\left\{B_{T}\right\}\right), \quad t \in[0, T]
$$


It is well known (see, e.g. [12]) that there exists a $\mathbb{G}$-Brownian motion $W$ such that if we define $Z$ with respect to this specific $W$ and set

$$
H_{t}:=\int_{0}^{t} \frac{B_{T}-B_{s}}{T-s} \mathrm{~d} s, \quad k_{t}:=1, \quad t \in[0, T],
$$

then $Z=B$, which clearly does not have $\mathbb{G}$-CFS.

Intuitively, when $H$ and $k$ depend on $W$, even though $k$ is always positive, they may use 'data' from $W$ to 'steer' the process $Z$ away from some regions of the path space, so that CFS does not hold.

Theorem 3.2. (CFS.) Let $\left(X_{t}\right)_{t \in[0, T]}$ be a continuous process, let $\left(W_{t}\right)_{t \in[0, T]}$ be a Brownian motion, let $\phi$ and $\psi$ be progressive functions from $[0, T] \times C([0, T])^{2}$ to $\mathbb{R}$, and let $\xi$ be a random variable. Define

$$
h_{t}:=\phi(t, W, X), \quad k_{t}:=\psi(t, W, X), \quad \mathcal{F}_{t}:=\sigma\left\{\xi, W_{s}, X_{s}: s \in[0, t]\right\}, \quad t \in[0, T] .
$$

If $W$ is a Brownian motion also with respect to $\mathbb{F}=\left(\mathcal{F}_{t}\right)_{t \in[0, T]}$,

$$
\mathrm{E}\left[\exp \left(r \int_{0}^{T} k_{s}^{-2} \mathrm{~d} s\right)\right]<\infty \text { for all } r>0, \quad \mathrm{E}\left[\exp \left(2 \int_{0}^{T} k_{s}^{-2} h_{s}^{2} \mathrm{~d} s\right)\right]<\infty,
$$

and

$$
\int_{0}^{T} k_{s}^{2} \mathrm{~d} s \leq \bar{K} \quad \text { a.s. for some constant } \bar{K} \in(0, \infty),
$$

then the process

$$
Z_{t}:=\xi+\int_{0}^{t} h_{s} \mathrm{~d} s+\int_{0}^{t} k_{s} \mathrm{~d} W_{s}, \quad t \in[0, T]
$$

has CFS.

Remark 3.3. In light of Examples 3.1 and 3.2, condition (3.4) seems nearly optimal. However, it is not that clear how necessary condition (3.5) is, i.e. if some nonuniform bound would suffice. That said, for the method we use in the present proof, this uniform bound appears to be unavoidable.

Proof of Theorem 3.2. For technical reasons, we assume without loss of generality that $W$, $X$, and $\mathbb{F}$ are defined on the whole interval $[0, \infty)$. In order to be able to use regular conditional laws, we define the underlying probability space as follows:

$$
\Omega:=C\left([0, \infty), \mathbb{R}^{2}\right) \times \mathbb{R}, \quad \mathcal{F}:=\mathscr{B}\left(C\left([0, \infty), \mathbb{R}^{2}\right) \times \mathbb{R}\right), \quad \mathrm{P}:=\operatorname{law}[(W, X), \xi],
$$

that is, for any $\omega=\left(\left(\omega_{1}^{\prime}, \omega_{2}^{\prime}\right), \omega^{\prime \prime}\right) \in \Omega$, we set $W_{t}(\omega):=\omega_{1}^{\prime}(t), X_{t}(\omega):=\omega_{2}^{\prime}(t)$ for all $t \in[0, \infty)$, and $\xi(\omega):=\omega^{\prime \prime}$. Furthermore, let us fix $\underline{t} \in[0, T), f \in C_{0}([\underline{t}, \infty))$ such that $f$ is absolutely continuous with bounded Radon-Nikodým derivative $f^{\prime}$ that satisfies $\operatorname{supp}\left(f^{\prime}\right) \subset[\underline{t}, T]$, and $\varepsilon>0$. Since $\Omega$ is a Polish space, by well-known results (see, e.g. Theorem II.89.1 of [18]), there exists a regular conditional law $\mu:=\mathrm{P}\left[\cdot \mid \mathcal{F}_{\underline{t}}\right]$ (a random measure on $(\Omega, \mathcal{F}))$. 
Step 1: conditioning. We are about to fix a realization of the random measure $\mu$, drawn from a set with probability 1 , under which $\hat{W}^{\underline{t}}$ is a Brownian motion with respect to $\left(\mathcal{F}_{t}\right)_{t \in[t, \infty)}$. To this end, for any $d \in \mathbb{Z}_{+}, \boldsymbol{t}=\left(t_{1}, \ldots, t_{d}\right) \in[0, \infty)^{d}$, and $\boldsymbol{q}=\left(q_{1}, \ldots, q_{d}\right) \in \mathbb{R}^{d}$, write

$$
\begin{aligned}
A_{1}(\boldsymbol{t}, \boldsymbol{q}) & :=\left\{W_{t_{1}} \leq q_{1}, \ldots, W_{t_{d}} \leq q_{d}\right\}, \\
A_{2}(\boldsymbol{t}, \boldsymbol{q}) & :=\left\{\hat{W}_{t_{1}}^{\underline{t}} \leq q_{1}, \ldots, \hat{W}_{t_{d}}^{t} \leq q_{d}\right\} \quad\left(\text { where } \boldsymbol{t} \in[\underline{t}, \infty)^{d}\right), \\
B(\boldsymbol{t}, \boldsymbol{q}) & :=\left\{X_{t_{1}} \leq q_{1}, \ldots, X_{t_{d-1}} \leq q_{d-1}, \xi \leq q_{d}\right\},
\end{aligned}
$$

and, for any $t \in[\underline{t}, \infty)$,

$$
\begin{aligned}
\mathcal{I} & :=\left\{A_{1}(\boldsymbol{t}, \boldsymbol{q}): d \in \mathbb{Z}_{+}, \boldsymbol{t} \in[0, \underline{t}]^{d} \cap(\mathbb{Q} \cup\{\underline{t}\})^{d}, \boldsymbol{q} \in \mathbb{Q}^{d}\right\}, \\
\mathcal{g}_{t} & :=\left\{A_{2}(\boldsymbol{t}, \boldsymbol{q}): d \in \mathbb{Z}_{+}, \boldsymbol{t} \in[\underline{t}, t]^{d} \cap(\mathbb{Q} \cup\{\underline{t}\})^{d}, \boldsymbol{q} \in \mathbb{Q}^{d}\right\}, \\
\mathcal{K}_{t} & :=\left\{B(\boldsymbol{t}, \boldsymbol{q}): d \in \mathbb{Z}_{+}, \boldsymbol{t} \in[0, t]^{d} \cap(\mathbb{Q} \cup\{\underline{t}\})^{d}, \boldsymbol{q} \in \mathbb{Q}^{d}\right\} .
\end{aligned}
$$

Furthermore, we note that

$$
\mathcal{C}_{t}:=\left\{A_{1} \cap A_{2} \cap B: A_{1} \in \mathcal{I}, A_{2} \in \mathcal{g}_{t}, B \in \mathcal{K}_{t}\right\}, \quad \mathscr{g}_{\infty}:=\bigcup_{t \in[\underline{t}, \infty)} \mathscr{g}_{t}
$$

are countable $\pi$-systems, and that, by continuity of the associated processes, $\sigma\left(\mathcal{C}_{t}\right)=\mathscr{F}_{t}, t \in$ $[\underline{t}, \infty)$, and $\tilde{\mathcal{F}}_{\infty} \hat{W}^{t}=\sigma\left(\mathcal{g}_{\infty}\right)$, respectively. Next, define sets

$$
\begin{aligned}
& E:=\bigcap_{A \in \mathscr{Z}_{\infty}}\{\omega \in \Omega: \mu(\omega, A)=\mathrm{P}[A]\}, \\
& F:=\bigcap_{\substack{t \in[\underline{t}, \infty) \cap \mathbb{Q}) \cap \\
s \in \mathbb{Q}_{+}}} \bigcap_{C \in \mathcal{C}_{t}}\left\{\omega \in \Omega: \int_{C} \mu\left(\omega, \mathrm{d} \omega^{\prime}\right)\left(W_{s+t}\left(\omega^{\prime}\right)-W_{t}\left(\omega^{\prime}\right)\right)=0\right\}, \\
& G_{1}:=\left\{\omega \in \Omega: Y_{1}(\omega):=\int_{\Omega} \mu\left(\omega, \mathrm{d} \omega^{\prime}\right) \exp \left(2\left\|f^{\prime}\right\|_{\infty}^{2} \int_{\underline{t}}^{T} k_{s}^{-2}\left(\omega^{\prime}\right) \mathrm{d} s\right)<\infty\right\}, \\
& G_{2}:=\left\{\omega \in \Omega: Y_{2}(\omega):=\int_{\Omega} \mu\left(\omega, \mathrm{d} \omega^{\prime}\right) \exp \left(2 \int_{\underline{t}}^{T} k_{s}^{-2}\left(\omega^{\prime}\right) h_{s}^{2}\left(\omega^{\prime}\right) \mathrm{d} s\right)<\infty\right\}, \\
& H:=\left\{\omega \in \Omega: \mu\left(\omega,\left\{\int_{0}^{T} k_{s}^{2} \mathrm{~d} s \leq \bar{K}\right\}\right)=1\right\} .
\end{aligned}
$$

It follows, from the assumption that $W$ is a Brownian motion with respect to $\mathbb{F}$, conditions (3.4) and (3.5), and from the disintegration theorem (see Theorem 6.4 of $[14]$ ), that $\mathrm{P}[E]=\mathrm{P}\left[G_{1}\right]=$ $\mathrm{P}\left[G_{2}\right]=\mathrm{P}[H]=1$. To check that $\mathrm{P}[F]=1$, note that the intersections in the definition of $F$ are countable, and that

$$
\mathrm{E}\left[\mathbf{1}_{C}\left(W_{s+t}-W_{t}\right) \mid \mathscr{F}_{\underline{t}}\right]=\mathrm{E}\left[\mathbf{1}_{C} \mathrm{E}\left[W_{s+t}-W_{t} \mid \mathscr{F}_{t}\right] \mid \mathscr{F}_{\underline{t}}\right]=0 \quad \text { P-a.s. }
$$

and use the disintegration theorem again.

For the remainder of the proof, we fix $\omega^{\star} \in E \cap F \cap G_{1} \cap G_{2} \cap H$, denote by $\left(\mathcal{F}^{\star}, \mathrm{P}^{\star}\right)$ the completion of $\left(\mathcal{F}, \mu\left(\omega^{\star}, \cdot\right)\right)$, and let $\mathbb{F}^{\star}=\left(\mathcal{F}_{t}^{\star}\right)_{t \in[t, \infty)}$ be the usual $\mathrm{P}^{\star}$-augmentation of $\left(\mathcal{F}_{t}\right)_{t \in[\underline{t}, \infty)}$. Again, denote by $\mathrm{E}^{\star}$ the expectation with respect to $\mathrm{P}^{\star}$. Since $\mathcal{F}_{\infty}$ is a $\pi$-system that generates $\tilde{\mathcal{F}}_{\infty}^{\hat{W}^{t}}$, it follows that $W^{\star}:=\hat{W}^{t}$ is a Brownian motion under $\mathrm{P}^{\star}$. To complete 
the first step, we still need to show that $W^{\star}$ is an $\left(\mathbb{F}^{\star}, \mathrm{P}^{\star}\right)$-martingale. We note that, for any $t \in[\underline{t}, \infty) \cap \mathbb{Q} \cup\{\underline{t}\}$ and $s \in \mathbb{Q}_{+}$,

$$
\mathbb{H}_{s, t}:=\left\{Y \in L^{\infty}\left(\Omega, \mathrm{P}^{\star}\right): \mathrm{E}^{\star}\left[Y\left(W_{s+t}-W_{t}\right)\right]=0\right\}
$$

is a vector space, closed with respect to uniform convergence, that contains all constant functions and all limits of uniformly bounded increasing sequences of elements of $\mathbb{H}_{s, t}$. Moreover, $\left\{\mathbf{1}_{C}: C \in \mathcal{C}_{t}\right\}$ is closed under multiplication (since $\mathcal{C}_{t}$ is a $\pi$-system), and since $\omega^{\star} \in F$, we have $\left\{\mathbf{1}_{C}: C \in \mathcal{C}_{t}\right\} \subset \mathbb{H}_{s, t}$. Hence, by the functional monotone class theorem (see, e.g. Theorem I.21 of [7]), $\mathbb{H}_{s, t}$ contains all bounded $\mathcal{F}_{t}$-measurable random variables. Thus, we find that

$$
\mathrm{E}^{\star}\left[W_{s+t}^{\star} \mid \mathcal{F}_{t}\right]=W_{t}^{\star} \quad \text { for any } t \in[\underline{t}, \infty) \cap \mathbb{Q} \text { and } s \in \mathbb{Q}_{+} .
$$

Now, let $t^{\prime}, t^{\prime \prime} \in[\underline{t}, \infty)$ be such that $t^{\prime \prime}>t^{\prime}$, and let $\left(t_{n}^{\prime \prime}\right) \subset\left[t, t^{\prime \prime}\right) \cap \mathbb{Q}$ and $\left(t_{n}^{\prime}\right) \subset\left[\underline{t}, t^{\prime}\right) \cap \mathbb{Q} \cup\{\underline{t}\}$ be such that $t_{n}^{\prime \prime} \uparrow t^{\prime \prime}$ and $t_{n}^{\prime} \uparrow t^{\prime}$, respectively, when $n \uparrow \infty$. Since, by continuity of $X$ and $W$,

$$
\mathcal{F}_{t^{\prime}}=\bigvee_{m=1}^{\infty} \mathcal{F}_{t_{m}^{\prime}}
$$

we have, by Theorem 7.23 of [14] and the continuity of $W^{\star}$,

$$
\mathrm{E}^{\star}\left[W_{t_{n}^{\prime \prime}}^{\star} \mid \mathcal{F}_{t^{\prime}}\right]=\lim _{m \rightarrow \infty} E^{\star}\left[W_{t_{n}^{\prime \prime}}^{\star} \mid \mathcal{F}_{t_{m}^{\prime}}\right]=\lim _{m \rightarrow \infty} W_{t_{m}^{\prime}}^{\star}=W_{t^{\prime}}^{\star} \quad \mathrm{P}^{\star} \text {-a.s. }
$$

Moreover, since $\mathrm{E}^{\star}\left[\sup _{n \in \mathbb{N}}\left|W_{t_{n}^{\prime \prime}}^{\star}\right|\right]<\infty$, the dominated convergence theorem for conditional expectations implies that

$$
\mathrm{E}^{\star}\left[W_{t^{\prime \prime}}^{\star} \mid \mathcal{F}_{t^{\prime}}\right]=\lim _{n \rightarrow \infty} \mathrm{E}^{\star}\left[W_{t_{n}^{\prime \prime}}^{\star} \mid \mathcal{F}_{t^{\prime}}\right]=W_{t^{\prime}}^{\star} \quad \mathrm{P}^{\star} \text {-a.s. }
$$

It follows now from standard results (see, e.g. Lemma II.72.2 of [18]) that $W^{\star}$ is an $\left(\mathbb{F}^{\star}, \mathrm{P}^{\star}\right)$ martingale.

Step 2: characterization of the support. To complete the proof, we shall now show that the law of $\hat{Z}^{t}$ under the measure $\mathrm{P}^{\star}$ is supported on $C_{0}([\underline{t}, T])$. To this end, we adapt the method employed in the proof of Lemma 3.1 of [20]. For the convenience of the reader, we do this in detail. Let us define

$$
Z_{t}^{\star}:=\int_{\underline{t}}^{t} \hat{k}_{s} \mathrm{~d} W_{s}^{\star}-\left\langle L, \int_{\underline{t}}^{\cdot} \hat{k}_{s} \mathrm{~d} W_{s}^{\star}\right\rangle_{t}, \quad t \in[\underline{t}, \infty),
$$

where

$$
\hat{k}_{t}:=k_{t} \mathbf{1}_{[\underline{t}, T]}(t)+\mathbf{1}_{(T, \infty)}(t), \quad L_{t}:=\int_{\underline{t}}^{t} \hat{k}_{s}^{-1}\left(f^{\prime}(s)-h_{s} \mathbf{1}_{[\underline{t}, T]}(s)\right) \mathrm{d} W_{s}^{\star} .
$$

We can easily check that

$$
Z_{t}^{\star}=Z_{t}-Z_{\underline{t}}-f(t), \quad t \in[\underline{t}, T] .
$$

Furthermore, we have, for all $t \in[\underline{t}, \infty)$, the uniform bound

$$
\langle L, L\rangle_{t}=\int_{\underline{t}}^{t} \hat{k}_{s}^{-2}\left(f^{\prime}(s)-h_{s} \mathbf{1}_{[\underline{t}, T]}(s)\right)^{2} \mathrm{~d} s \leq 2\left(\left\|f^{\prime}\right\|_{\infty}^{2} \int_{\underline{t}}^{T} k_{s}^{-2} \mathrm{~d} s+\int_{\underline{t}}^{T} k_{s}^{-2} h_{s}^{2} \mathrm{~d} s\right),
$$


using which we obtain, by the Cauchy-Schwarz inequality,

$$
\mathrm{E}^{\star}\left[\mathrm{e}^{\langle L, L\rangle_{\infty} / 2}\right] \leq \sqrt{Y_{1}\left(\omega^{\star}\right) Y_{2}\left(\omega^{\star}\right)}<\infty
$$

Consequently, Novikov's criterion implies that the Doléans exponential

$$
\mathcal{E}(L)_{t}:=\mathrm{e}^{L_{t}-\langle L, L\rangle_{t} / 2}, \quad t \in[\underline{t}, \infty),
$$

is a uniformly integrable $\left(\mathbb{F}^{\star}, \mathrm{P}^{\star}\right)$-martingale, closable at $\infty$. Hence, we may define a new probability measure $\mathrm{Q}^{\star} \sim \mathrm{P}^{\star}$ by $\mathrm{d}^{\star}:=\mathcal{E}(L)_{\infty} \mathrm{dP}^{\star}$.

Now, since $\int_{\underline{t}}^{\cdot} \hat{k}_{s} \mathrm{~d} W_{s}^{\star}$ is an $\left(\mathbb{F}^{\star}, \mathrm{P}^{\star}\right)$-martingale, by Girsanov's theorem, $Z^{\star}$ is an $\left(\mathbb{F}^{\star}, \mathrm{Q}^{\star}\right)$ martingale. Moreover, we have

$$
\left\langle Z^{\star}, Z^{\star}\right\rangle_{t}=\int_{\underline{t}}^{t} \hat{k}_{s}^{2} \mathrm{~d} s \geq(t-T)_{+} \rightarrow \infty \quad \text { as } t \rightarrow \infty,
$$

so, by the Dambis, Dubins-Schwarz theorem, there exists a Brownian motion $\left(B_{t}\right)_{t \in[0, \infty)}$ under $\mathrm{Q}^{\star}$ such that $Z_{t}^{\star}=B_{\left\langle Z^{\star}, Z^{\star}\right\rangle_{t}}, t \in[\underline{t}, \infty), \mathrm{Q}^{\star}$-a.s. Thus, we have

$$
\mathrm{Q}^{\star}\left[\sup _{t \in[\underline{t}, T]}\left|Z_{t}^{\star}\right|<\varepsilon\right]=\mathrm{Q}^{\star}\left[\sup _{t \in[\underline{t}, T]}\left|B_{\left\langle Z^{\star}, Z^{\star}\right\rangle_{t}}\right|<\varepsilon\right] \geq \mathrm{Q}^{\star}\left[\sup _{u \in[0, \bar{K}]}\left|B_{u}\right|<\varepsilon\right]>0,
$$

since $\left\langle Z^{\star}, Z^{\star}\right\rangle_{t} \leq \bar{K}$ for all $t \in[\underline{t}, T]$, and since the Wiener measure is supported on $C_{0}([0, \bar{K}])$ (see, e.g. Corollary VIII.2.3 of [17]). By the equivalence of the measures we may substitute $\mathrm{Q}^{\star}$ for $\mathrm{P}^{\star}$ in (3.7). Using the fact that $\mathrm{P}^{\star}$ coincides with $\mu\left(\omega^{\star}, \cdot\right)$ on $\mathcal{F}$ and

$$
B^{\prime}:=\left\{\sup _{t \in[\underline{t}, T]}\left|Z_{t}-Z_{\underline{t}}-f(t)\right|<\varepsilon\right\} \in \mathcal{F},
$$

by (3.6) we have $\mu\left(\omega^{\star}, B^{\prime}\right)>0$. To conclude, note that functions having the properties of $f$ are dense in $C_{0}([\underline{t}, T])$, so $Z$ has $\mathbb{F}$-CFS by Lemma 2.1. Finally, $Z$ has $\mathbb{F}^{Z}$-CFS by Corollary 2.1 and Lemma 2.3.

\subsection{Independent integrands and general integrators}

Since Brownian motion has CFS, one might wonder if the preceding results generalize to the case in which the integrator is merely a continuous process with CFS. While the proofs of these results rely on methods specific to Brownian motion (martingales, time changes), in the case of independent integrands of finite variation $\left(\mathrm{Z}^{3}\right)$ we are able to prove this conjecture.

Theorem 3.3. (CFS.) Let $\left(H_{t}\right)_{t \in[0, T]}$ be a continuous process, let $\left(k_{t}\right)_{t \in[0, T]}$ be a process of finite variation, and let $\left(X_{t}\right)_{t \in[0, T]}$ be a continuous process independent of $(H, k)$. If $X$ has CFS and

$$
\inf _{t \in[0, T]}\left|k_{t}\right|>0 \quad \text { a.s. }
$$

then the process

$$
Z_{t}:=H_{t}+\int_{0}^{t} k_{s} \mathrm{~d} X_{s}, \quad t \in[0, T]
$$

has CFS.

Remark 3.4. The stochastic integral in (3.9) exists as a pathwise Riemann-Stieltjes integral. This well-known fact follows from the (discrete) integration-by-parts formula. 
Proof of Theorem 3.3. First, denote by $\mathbb{G}=\left(g_{t}\right)_{t \in[0, T]}$ the filtration given by

$$
g_{t}:=\tilde{\mathcal{F}}_{t}^{X} \vee \sigma\left\{H_{s}, k_{s}: s \in[0, T]\right\},
$$

and let $\underline{t} \in[0, T), f \in C_{0}([\underline{t}, T])$, and $\varepsilon>0$. Furthermore, define

$$
g_{t}:=\int_{0}^{t} k_{s}^{-1} \mathrm{~d}\left(f(s)+H_{\underline{t}}-H_{s}\right), \quad t \in[\underline{t}, T]
$$

which is well defined since $\left(k_{t}^{-1}\right)_{t \in[t, T]}$ is of finite variation, by (3.8). Integration by parts and the Love-Young inequality yield, for all $t \in[\underline{t}, T]$,

$$
\begin{aligned}
\left|Z_{t}-Z_{\underline{t}}-f(t)\right| & =\left|\int_{\underline{t}}^{t} k_{s} \mathrm{~d}\left(X_{S}-X_{\underline{t}}-g_{s}\right)\right| \\
& =\left|k_{t}\left(X_{t}-X_{\underline{t}}-g_{t}\right)-\int_{\underline{t}}^{t}\left(X_{S}-X_{\underline{t}}-g_{s}\right) \mathrm{d} k_{s}\right| \\
& \leq \underbrace{\left(\sup _{t \in[\underline{t}, T]}\left|k_{S}\right|+\operatorname{TV}_{[\underline{t}, T]}(k)\right)}_{=: M(k)} \sup _{t \in[\underline{t}, T]}\left|X_{t}-X_{\underline{t}}-g_{t}\right|,
\end{aligned}
$$

where $\operatorname{TV}_{[\underline{t}, T]}(k)$ denotes the total variation of the path of $k$ on the interval $[\underline{t}, T]$. This estimate implies the inclusion

$$
\left\{\sup _{t \in[\underline{t}, T]}\left|X_{t}-X_{\underline{t}}-g_{t}\right|<\frac{\varepsilon}{2 M(k)}\right\} \subset\left\{\sup _{t \in[\underline{t}, T]}\left|Z_{t}-Z_{\underline{t}}-f(t)\right|<\varepsilon\right\} .
$$

Hence, by monotonicity of conditional expectations, and by Lemmas 2.1, 2.2, and 2.3, it suffices to show that

$$
\mathrm{E}\left[I\left(\hat{X}^{t}, g, \frac{\varepsilon}{2 M(k)}\right) \mid g_{\underline{t}}\right]>0 \text { a.s. }
$$

To this end, note that since $X$ is independent of $H$ and $k$, it has $\mathbb{G}$-CFS, and denote by $v$ some regular $g_{t}$-conditional law of $\hat{X}^{t}$. The disintegration theorem (see Theorem 6.4 of [14]) now yields

$$
\mathrm{E}\left[I\left(\hat{X}^{t}, g, \frac{\varepsilon}{2 M(k)}\right) \mid g_{\underline{t}}\right]=\int_{C([\underline{t}, T])} \mathbf{1}_{B(g, \varepsilon /(2 M(k)))}(x) v(\cdot, \mathrm{d} x)>0 \quad \text { a.s. }
$$

since $X$ has $\mathbb{G}-\mathrm{CFS}$.

\section{Applications}

In this section we establish CFS for certain price processes used in mathematical finance. The main motivation for these applications stems from the desire to uncover more concrete price processes to which the superreplication result in [10] applies.

\subsection{Stochastic volatility models}

Let us consider a price process $\left(P_{t}\right)_{t \in[0, T]}$ in $\mathbb{R}_{+}$defined by

$$
\mathrm{d} P_{t}=P_{t}\left(f\left(t, V_{t}\right) \mathrm{d} t+\rho g\left(t, V_{t}\right) \mathrm{d} B_{t}+\sqrt{1-\rho^{2}} g\left(t, V_{t}\right) \mathrm{d} W_{t}\right), \quad P_{0}=p_{0} \in \mathbb{R}_{+},
$$

where $f, g \in C\left([0, T] \times \mathbb{R}^{d}\right), \rho \in(-1,1),(W, B)$ is a planar Brownian motion, and $V$ is 
a process in $\mathbb{R}^{d}$ such that $g\left(t, V_{t}\right)>0$ a.s. for all $t \in[0, T]$. Furthermore, we assume that $(B, V)$ is independent of $W$. We may now verify that $P$ has CFS, since by positivity of $P$, Itô's formula yields

$$
\begin{aligned}
\log P_{t}= & \log p_{0}+\int_{0}^{t}\left(f\left(s, V_{s}\right)-\frac{1}{2} g\left(s, V_{s}\right)^{2}\right) \mathrm{d} s+\rho \int_{0}^{t} g\left(s, V_{s}\right) \mathrm{d} B_{s} \\
& +\sqrt{1-\rho^{2}} \int_{0}^{t} g\left(s, V_{s}\right) \mathrm{d} W_{s},
\end{aligned}
$$

which clearly satisfies the assumptions of Theorem 3.1 (see Remark 3.1), and finally we may invoke (2.1).

Let us briefly review some well-known special cases of (4.1). In standard Markovian stochastic volatility models, the process $V$ is a one-dimensional diffusion driven by $B$, that is,

$$
\mathrm{d} V_{t}=\alpha\left(t, V_{t}\right) \mathrm{d} t+\beta\left(t, V_{t}\right) \mathrm{d} B_{t}, \quad V_{0}=v_{0} \in \mathbb{R} .
$$

The popular models introduced by Heston (with leverage effects when $-1<\rho<0$ ); Hull and White; E. M. Stein, J. C. Stein and Scott; and Wiggins are special cases of (4.1); see, e.g. [8] for details. One notable special case of (4.1), in which $V$ is not Markovian, is the model of Comte and Renault [5], which was designed to capture long-memory effects in volatility. It can be obtained by setting $g(t, v):=\mathrm{e}^{v}, \rho:=0$, and choosing $V$ to be a fractional Ornstein-Uhlenbeck process independent of $W$.

Special cases of (4.1) in which volatility may jump include the stochastic volatility model of Barndorff-Nielsen and Shephard [1] and the regime switching model of Guo [11]. To see why in the former model we have $g\left(t, V_{t}\right)>0$ a.s. for all $t \in[0, T]$, recall that we obtain the model by specifying

$$
V_{t}:=\int_{-\infty}^{t} \mathrm{e}^{-r(t-s)} \mathrm{d} L_{r s}, \quad t \in[0, T],
$$

where $\left(L_{t}\right)_{t \in \mathbb{R}}$ is an increasing Lévy process (a subordinator) without drift, such that $L_{0}=0$ a.s., independent of $W, r>0, \rho:=0$, and $g(t, v):=\sqrt{v}$. To exclude the uninteresting case with zero volatility (when $P$ of course does not have CFS), let us assume that $\mathrm{P}\left[L_{1}=0\right]<1$. We find that $V_{t} \geq \mathrm{e}^{-r T} V_{0}$ for all $t \in[0, T]$ since $L$ is increasing. Furthermore, using stationarity and independence of the increments of $L$, we obtain, for all $k \in \mathbb{Z}_{+}$,

$$
\mathrm{P}\left[V_{0}=0\right] \leq \mathrm{P}\left[L_{-i+1}-L_{-i}=0 \text { for all } i=1, \ldots, k\right]=\mathrm{P}\left[L_{1}=0\right]^{k},
$$

and letting $k \rightarrow \infty$ we find that $V_{0}>0$ a.s., from which the desired property follows.

Remark 4.1. The absolute continuity of the drift of $P$ is of course not necessary. Namely, we can easily establish CFS similarly e.g. for any price process $P_{t}:=\mathrm{e}^{f(t)+(g(V) \cdot W)_{t}}, t \in[0, T]$, where $f$ is an arbitrary continuous function. For certain choices of $f$, process $P$ is known to admit arbitrage opportunities in frictionless pricing models (see [6]).

\subsection{Stochastic differential equations}

Finally, let us consider a price process $\left(P_{t}\right)_{t \in[0, T]}$ in $\mathbb{R}_{+}$given by the stochastic differential equation

$$
\mathrm{d} P_{t}=\mu(t, P) \mathrm{d} t+\sigma(t, P) \mathrm{d} W_{t}, \quad P_{0}=p_{0} \in \mathbb{R}_{+},
$$


where $\mu$ and $\sigma$ are progressive functions such that, for some constants $\bar{\mu}>0$ and $\bar{\sigma}>1$,

$$
\begin{gathered}
|\mu(t, x)| \leq \bar{\mu} x(t), \quad \bar{\sigma}^{-1} x(t) \leq|\sigma(t, x)| \leq \bar{\sigma} x(t), \\
x \in C_{p_{0}}\left([0, T], \mathbb{R}_{+}\right), \quad t \in[0, T] .
\end{gathered}
$$

Furthermore, we assume that $\mu$ and $\sigma$ are such that (4.2) has a weak solution, which, by definition, means that there exist a filtration $\mathbb{F}=\left(\mathcal{F}_{t}\right)_{t \in[0, T]}$ and $\mathbb{F}$-adapted continuous processes $P$ and $W$ that solve (4.2), such that $W$ is an $\mathbb{F}$-Brownian motion. Clearly, we may assume that $\mathcal{F}_{t}=\sigma\left\{P_{s}, W_{s}: s \in[0, t]\right\}$. Now, since $P$ is positive, Itô's formula yields

$$
\log P_{t}=\log p_{0}+\int_{0}^{t}\left(\frac{\mu(s, P)}{P_{s}}-\frac{\sigma^{2}(s, P)}{2 P_{s}^{2}}\right) \mathrm{d} s+\int_{0}^{T} \frac{\sigma(s, P)}{P_{S}} \mathrm{~d} W_{s} .
$$

Setting $X:=P$ and noting that (4.3) implies the conditions of Theorem 3.2, by (2.1), it follows that $P$ has $\mathrm{CFS}$.

\section{Acknowledgements}

I would like to thank Tommi Sottinen for introducing me to the topic and for helpful discussions. I also thank Esa Nummelin for reading previous drafts of this paper and for constant encouragement. Finally, I thank Boualem Djehiche, Dario Gasbarra, and Esko Valkeila for valuable comments. Research was supported by the Academy of Finland (project 116747) and the Finnish Cultural Foundation.

\section{References}

[1] Barndorff-Nielsen, O. E. And Shephard, N. (2001). Non-Gaussian Ornstein-Uhlenbeck-based models and some of their uses in financial economics. J. R. Statist. Soc. Ser. B 63, 167-241.

[2] Bender, C., Sottinen, T. and Valkeila, E. (2008). Pricing by hedging and no-arbitrage beyond semimartingales. Finance Stoch. 12, 441-468.

[3] Cheridito, P. (2001). Mixed fractional Brownian motion. Bernoulli 7, 913-934.

[4] Cherny, A. (2008). Brownian moving averages have conditional full support. Ann. Appl. Prob. 18, 1825-1830.

[5] Comte, F. and Renault, E. (1998). Long memory in continuous-time stochastic volatility models. Math. Finance 8, 291-323.

[6] Delbaen, F. And Schachermayer, W. (1995). The existence of absolutely continuous local martingale measures. Ann. Appl. Prob. 5, 926-945.

[7] Dellacherie, C. and Meyer, P.-A. (1975). Probabilités et Potentiel. Hermann, Paris.

[8] Frey, R. (1997). Derivative asset analysis in models with level-dependent and stochastic volatility. CWI Quart. 10, $1-34$.

[9] Gasbarra, D., Sottinen, T. and van Zanten, H. (2008). Conditional full support of Gaussian processes with stationary increments. Preprint 487, Department of Mathematics and Statistics, University of Helsinki.

[10] Guasoni, P., Rásonyi, M. and Schachermayer, W. (2008). Consistent price systems and face-lifting pricing under transaction costs. Ann. Appl. Prob. 18, 491-520.

[11] Guo, X. (2001). An explicit solution to an optimal stopping problem with regime switching. J. Appl. Prob. 38, 464-481.

[12] Jeulin, T. And Yor, M. (1979). Inégalité de Hardy, semimartingales, et faux-amis. In Séminaire de Probabilités XIII (Lecture Notes Math. 721), Springer, Berlin, pp. 332-359.

[13] Kabanov, Y. and Stricker, C. (2008). On martingale selectors of cone-valued processes. In Séminaire de Probabilités XLI (Lecture Notes Math. 1934), Springer, Berlin, pp. 439-442.

[14] Kallenberg, O. (2002). Foundations of Modern Probability, 2nd edn. Springer, New York.

[15] Kallianpur, G. (1971). Abstract Wiener processes and their reproducing kernel Hilbert spaces. Z. Wahrscheinlichkeitsth. 17, 113-123.

[16] Millet, A. ANd Sanz-Solé, M. (1994). A simple proof of the support theorem for diffusion processes. In Séminaire de Probabilités XXVIII (Lecture Notes Math. 1583), Springer, Berlin, pp. 36-48.

[17] Revuz, D. And Yor, M. (1999). Continuous Martingales and Brownian Motion, 3rd edn. Springer, Berlin. 
[18] Rogers, L. C. G. And Williams, D. (2000). Diffusions, Markov Processes, and Martingales, Vol. 2. Cambridge University Press.

[19] Stroock, D. W. (1971). On the growth of stochastic integrals. Z. Wahrscheinlichkeitsth. 18, 340-344.

[20] Stroock, D. W. and Varadhan, S. R. S. (1972). On the support of diffusion processes with applications to the strong maximum principle. In Proc. 6th Berkeley Symp. Mathematical Statistics and Probability, Vol. III, California Press, Berkeley, CA, pp. 333-359. 